\title{
Ordenamiento territorial en Chile: nuevo escenario para la gobernanza regional ${ }^{1}$
}

\section{Land planning in Chile: new scenario for regional governance}

\author{
Arturo Orellana Ossandón² [0, Federico Arenas Vásquez³ \\ y Daniel Moreno Alba ${ }^{4}$
}

\begin{abstract}
RESUMEN
A partir de un análisis de contexto sobre la evolución de los paradigmas en torno al ordenamiento territorial desde una perspectiva reciente, este trabajo se enfoca en describir y analizar el proceso político, normativo e institucional en términos de su potencial incidencia en el ordenamiento territorial para las regiones desde un enfoque interpretativo. En particular, se toma en consideración las implicancias de la Ley 21.074 en los diversos instrumentos de planificación a escala intercomunal o regional sobre la base de algunos criterios que están relacionados con la descentralización política, administrativa y fiscal, para lo cual previamente se precisan algunos antecedentes históricos que preceden al nuevo escenario para el ejercicio de la gobernanza regional en materia de ordenamiento territorial. En síntesis, este trabajo demuestra que las posibilidades que las regiones ejerzan una gobernanza en propiedad en materia de ordenamiento territorial, resultan ser solo mínimas en términos de su incidencia, incluso con la existencia y aprobación de un Plan Regional de Ordenamiento Territorial.
\end{abstract}

Palabras clave: Regionalización, descentralización, gobernanza, ordenamiento territorial, instrumentos de planificación.

\begin{abstract}
Based on a context analysis on the evolution of paradigms around land planning from a recent perspective, this work focuses on describing and analyzing the political, regulatory and institutional process in terms of its potential impact on land planning for regions from an interpretive approach. In particular, the implications of Law 21.074 are taken into consideration in the various planning instruments at the intercommunal or regional level based on some criteria that are related to political, administrative and fiscal decentralization, for which previously some historical antecedents are specified that precede the new scenario for the exercise of regional governance in matters of land planning. In summary, this work demonstrates that the possibilities for the regions to exercise proprietary governance in matters of land planning, turn out to be only minimal in terms of their incidence, even with the existence and approval of a Regional Plan for Land Planning.
\end{abstract}

Keywords: Regionalization, decentralization, governance, land planning, planning instruments.

El presente manuscrito forma parte del Núcleo de Investigación sobre Gobernanza y Ordenamiento Territorial (NUGOT). Profesor asociado, Instituto de Estudios Urbanos y Territoriales, Pontificia Universidad Católica de Chile, Investigador del Núcleo sobre Gobernanza y Ordenamiento Territorial (NUGOT). Correo electrónico: amorella@uc.cl

Profesor titular, Director del Instituto de Geografía de la Pontificia Universidad Católica de Chile. Correo electrónico: farenasv@uc.cl

4 Pontificia Universidad Católica de Chile e investigador del Núcleo sobre Gobernanza y Ordenamiento Territorial (NUGOT).

Correo electrónico: dlmoreno@uc.cl 


\section{Estado del arte}

El siglo XXI está presentando cambios acelerados en cuanto a las visiones sobre el territorio, a la hora de planificar en occidente. Así entonces, mientras a partir de la gran crisis financiera del año 29 se impuso una visión espacial keynesiana, que perduró casi 40 años, donde se imponía la economía nacional y por ende la regulación por sobre los intereses regionales. A partir de la llamada crisis energética de comienzos de los años '70 emergió con fuerza el protagonismo de los gobiernos locales y acuerdos públicos-privados, dando inicio desde los años ' 80 al nuevo regionalismo que acentuaba la importancia de la gobernanza multinivel (Galland \& Harrison, 2019). A juicio de estos mismos autores, el desafío hoy está en generar una planificación en tiempo real, sobre la base de una estructura de gobernanza más flexible (ibid.), entendiendo las tendencias hacia la autonomización de las regiones como parte del proceso de reescalamiento que está experimentando el Estado (Fernández et al., 2008).

En tal sentido, se hace indispensable que las ciudades, regiones y Estados no operen como configuraciones geográficas mutuamente excluyentes o competitivas, sino como formas de organización territorial interdependientes, densamente superpuestas como plantea Brenner (1999). Por lo tanto, los desafíos de la regionalización precisan de una gobernanza territorial a escala regional sostenida en un modelo de desarrollo consensuado, pero también con atribuciones y competencias suficientes para planificar y gestionar, en forma integrada y articulada bajo un enfoque multiescalar (Rehren et al., 2018), particularmente necesario en cuanto a la interdependencia de sus instrumentos de ordenación y planificación del territorio.

Así entonces, el ordenamiento del territorio supone un ejercicio técnico con objetivos políticos, lo cual va desde la búsqueda del interés público hasta buscar superar fallas de mercado, para alcanzar un proyecto moderno de estado, sociedad y economía (Ferrao, 2011). Desde esta perspectiva, las políticas públicas en ordenamiento territorial resultan ser esenciales para transformar regiones, países o espacios transfronterizos, en lugares más atractivos, sustentables, desarrollados, justos y democráticos (ibid.).

Desde esa perspectiva, las experiencias internacionales respecto al debate sobre el derrotero que deben seguir las reformas en materia de ordenamiento territorial no resultan ser del todo consensuadas, advirtiendo que las implicancias para una mejor gobernanza multinivel van de la mano de la estructura de Estado que permita en materia de descentralización a escala subnacional y local (Cámara, 2018; Hernández, 2010; Sánchez et al., 2013). En tal sentido, las experiencias de España, Colombia y México dan cuenta que aún con matices, se apela a mayores grados de descentralización particularmente en lo relativo a la capacidad de los gobiernos subnacionales y locales de incidir en los instrumentos de planificación territorial.

En contraposición a ello, hoy los intentos unitarios, centralistas y sectoriales de ordenar el territorio para los desafíos del futuro resultan ser absolutamente inconcebibles desde el discurso político y, además, carentes de fundamento desde el punto de vista técnico (Arenas \& Orellana, 2019). Como señala Marshall (2018), Chile ha vivido un proceso tardío y fragmentado de la descentralización de la planificación territorial, en el sentido de un traspaso significativo de atribuciones, competencias y facultades de planificación a gobiernos regionales y municipalidades. 
Nuestro país, caracterizado por un paisaje rico y diverso geográficamente, presenta significativos desequilibrios en términos de su configuración sociodemográfica y en cuanto a su desarroIlo social, económico y ambiental, como gran parte los países latinoamericanos según se constata en el estudio sobre disparidades territoriales de la CEPAL (2017). Particularmente, en el caso de Chile variados trabajos publicados sobre calidad de vida así lo demuestran (Orellana et al., 2013; Orellana \& Marshall, 2017; Orellana \& Marshall, 2019), y aunque estos apuntan solo a un universo de 99 comunas de las 346 existentes en el país ${ }^{5}$, permiten establecer las importantes brechas que existen en términos de la dotación de bienes y servicios públicos y privados entre los núcleos capitales en regiones. Del mismo modo, los indicadores y estándares definidos por el SIEDU ${ }^{6}$ reafirman igualmente tales desigualdades en el país.

No existen dos diagnósticos respecto de las razones que sostienen estas disparidades, ya que fundamentalmente obedecen al hecho de un marco normativo-institucional que ha reforzado en casi dos siglos la existencia de un estado unitario, centralista y sectorializado. Así entonces, se refirma lo señalado por Amin y Thrift (1995), en el sentido que bajo estas circunstancias las regiones pasan a ser instancias receptoras de las dinámicas y decisiones provenientes de la "mano invisible" del Estado nacional, así como de la propia "mano invisible del mercado", como supuesta acción equilibradora del desarrollo territorial. En sintonía con lo anterior, Prats (2009), plantea que en este caso no solo se producen discriminaciones económicas y sociales, sino también culturales y territoriales. En la práctica, esto significa que cuando la gente de un lugar no tiene las mismas posibilidades de participar de la vida democrática (respecto a otros lugares) o cuando su identidad no es debidamente valorada y reconocida a nivel nacional, la democracia se deteriora y la ciudadanía se distancia o se pone al margen del sistema (Arenas \& Orellana, 2019), cuestión que debilita la gobernanza en materia de ordenación del territorio.

Desde la perspectiva de Rondinelli (1981), sigue siendo válido entonces que la descentralización facilita la articulación e implementación de las políticas de desarrollo diseñadas para lograr crecimiento con equidad, fortaleciendo la capacidad de las unidades regionales y subregionales permitiendo, además, capacitar y fortalecer los liderazgos políticos para que sean capaces de identificar sus propios problemas y prioridades de desarrollo a escala regional y local. No obstante, el resultado del proceso va a depender de la capacidad de los gobiernos para asegurar una efectiva coordinación intergubernamental, permitiendo conjugar de manera armónica e integral un adecuado reparto de atribuciones y responsabilidades entre las diferentes instancias del gobierno, asegurando el equilibrio macroeconómico y la búsqueda de mayor eficiencia y equidad en la asignación del gasto público (Aghón \& Cortés, 1998).

En el caso de nuestro país, como señala Marshall (2020), durante décadas hemos evidenciando un proceso dicotómico entre el desarrollo de los procesos legislativos de descentralización político-administrativa y aquellos de regulación del desarrollo urbano y planificación territorial. Por lo tanto, se hace indispensable una gobernanza que distinga las diversas escalas del territorio y las decisiones asociadas, propiciando un fortalecimiento de las capacidades y atribuciones

Hay que considerar que en estas 99 comunas con una población urbana que supera el $90 \%$ vive el $80 \%$ de la población de Chile

Sistema de Indicadores y Estándares del Desarrollo Urbano (SIEDU) administrado por el Instituto Nacional de Estadísticas (INE). Disponible en http://siedu.ine.cl/index.html 
locales y regionales en la gestión del territorio, así como la articulación institucional del Estado y de este con el sector privado productivo y la sociedad civil organizada (Arenas \& Orellana, 2019).

La Ley 21.074 constituye un avance significativo en dotar al país y a las regiones de una Política Nacional de Ordenamiento del Territorio (PNOT), que además articule una Política Nacional de Desarrollo Urbano (PNDU) hoy vigente, con una Política Nacional de Desarrollo Rural (PNDR) recientemente promulgada (5 de mayo de 2020). Sin embargo, resulta importante juzgar a la luz del nuevo escenario que plantea esta Ley, si el marco normativo-institucional que se plantea sobre la base de los instrumentos vigentes y el propio Plan Regional de Ordenamiento del Territorio (PROT), resulta suficiente para garantizar una buena gobernanza en materia de ordenación de territorio para los Gobiernos Regionales (GORE).

\section{Algunos antecedentes históricos para el caso de chile}

Uno de los rasgos más característicos de Chile es una gran diversidad geográfica explicada tanto por su extensión en latitud, su relieve y su posición en el continente sudamericano. La diversidad resultante de sus $4.230 \mathrm{~km}$ de extensión latitudinal (entre Arica y las Islas Diego Ramírez), contrasta con su ancho medio de $177 \mathrm{~km}$ en longitud, pero no con su variación en altitud, la que puede elevarse hasta los 6.893 metros como ocurre con el Nevado Ojos del Salado y a más de 5.000 metros, como ocurre con otras veinticinco cumbres (Cereceda \& Errázuriz, 2005), lo que se traduce en una diversidad climática, vegetacional y paisajística única.

Como se menciona en Arenas (2017), hay que reconocer que estos canales de expresión de la geografía chilena (o canales de influencia), representan innumerables ventajas, pero al mismo tiempo una serie de desafíos. Entre las ventajas están, sin duda, "la cercanía al mar desde casi todos los puntos del territorio chileno, la existencia de un clima con cuatro estaciones bien marcadas en una parte importante de Chile central, la existencia de la cordillera de Los Andes que actúa como un biombo climático, permitiendo la acumulación de nieve y de reservas de agua en la época de los deshielos, entre otras." Entre los principales desafíos aparece la necesidad de adaptarse a la mencionada influencia de la geografía, que se manifiesta "a través de distintos canales, incluyendo entre estos a las amenazas naturales" (Arenas, 2017).

Chile ha impulsado varias acciones que, desde mediados de los años 1960, incluyen la puesta en marcha de estrategias sucesivas en el plano de la organización y administración territorial: la estrategia denominada de desarrollo regional polarizado en primer lugar $y$, luego, una segunda basada en la idea del desarrollo regional integrado y, finalmente, la denominada regionalización. Entre las acciones previas a las tres estrategias mencionadas, estuvo la creación de zonas francas en las ciudades de Arica y Castro, "utilizadas como instrumentos para impulsar el desarrollo de ciertos puntos y por esa vía, la dinamización de sus espacios circundantes" (Arenas, 2009).

En el marco de las estrategias mencionadas, se puede destacar la idea de políticas diferenciadas en el territorio, especialmente la propuesta de una política específica para el sistema de asentamientos urbanos, definiendo funciones para los centros de distinto nivel y, además, intentando modificar las jerarquías preexistentes buscando reequilibrar dicho sistema y reducir la macrocefalia urbana (Arenas, 2009; 2017). 
Durante la denominada estrategia de regionalización, impulsada después del golpe de estado de 1973, se instala una nueva división político-administrativa, que divide al país en trece regiones y estas a su vez en provincias, buscando mejorar la administración y el desarrollo territorial del país. Tal como se señala en Arenas (2009; 2017), además, se desconcentra espacialmente la estructura del Estado, estableciendo las oficinas regionales de los servicios públicos y las denominadas secretarias regionales ministeriales.

Es recién a inicio de los años noventa, una vez recuperada la democracia, que se promulga en el año 1993 la Ley $19.175^{7}$ y se retoma la posibilidad de avanzar hacia una institucionalidad descentralizada. Aunque nunca se pudo poner en marcha una política nacional de desarrollo regional, si se reforzaron algunos instrumentos, como el Fondo Nacional de Desarrollo Regional (FNDR), creado durante la regionalización y que perdura, con modificaciones en su naturaleza y en su forma de distribución, hasta hoy.

Un balance rápido muestra que desde el año 1990 hasta el 2010, durante los veinte años de gobierno de la Concertación de Partidos por la Democracia, hubo avances importantes, tales como, la instalación de los Gobiernos Regionales (GORE), el aumento de la inversión pública de decisión regional, avances en desconcentración de las decisiones hacia las estructuras regionales y locales y, la creación de dos nuevas regiones en el año 2007 (de Los Ríos y de Arica y Parinacota). Sin embargo, como se señala en Arenas (2017), "la institucionalidad pública regional no logra articularse y aparece atomizada en lógicas particulares que se anteponen a la lógica territorial, con un sector público que se coordina poco entre sí y menos con un sector privado que, salvo excepciones, se encuentra poco comprometido con los temas y desafíos relacionados al desarrollo regional". Más aún, un reporte publicado la OCDE-SUBDERE (2017) señala que en Chile las estrategias, políticas e inversiones territoriales tienden a ser definidas centralmente y a estar fragmentadas por sector.

Entre 2010 y 2018, durante las administraciones de la Alianza por Chile (entre 2010-2014) y Nueva Mayoría (entre 2014-2018), el proceso de descentralización y de mejora de la administración y gestión territorial ha estado prácticamente estancado, con la excepción de la modificación que permitió elegir por votación directa a los Consejeros Regionales (promulgada en 2013), y la creación de la Región de Ñuble en 2017, que entró en vigencia en septiembre de 2018. Sin embargo, como se menciona en Arenas (2017), la modificación de la estructura político-administrativa chilena no es una novedad y parecería no garantizar nada concreto si se miran los constantes cambios que se han llevado a cabo a través de la historia, y que se reflejan en el pasado en numerosas readecuaciones (Sagredo et al., 2016).

Aunque finalmente se modificó la Ley Orgánica Constitucional de Gobierno y Administración Regional en enero de 2018 y que permitirá la elección directa de los nuevos Gobernadores Regionales en principio en 2021, lamentablemente el trabajo de definición de una Política Nacional de Ordenamiento Territorial desarrollado durante todo el 2017 no se tradujo en su aprobación hasta ahora. Hubiese significado un reimpulso al proceso de descentralización y de ordenamiento territorial, a través de la existencia efectiva del Plan Regional de Ordenamiento Territorial (PROT), dentro de las atribuciones de planificación territorial que empiezan a consolidarse en el seno de 
los gobiernos regionales, sin embargo, la administración de Chile Vamos que asumió en marzo de 2018, la retiró de la Contraloría General de la República.

Finalmente, la tarea de los GORE en materia territorial se verá reforzado una vez que se defina la política de ordenamiento territorial ya mencionada al final del acápite precedente y se le pueda dar sustento legal a los PROT, avanzado hacia la instalación de una mirada territorial (transversal) que haga contrapeso a la mirada sectorial, y una mirada de escala regional/comunal que complemente y corrija la mirada nacional desprovista de sensibilidad por la diversidad geográfica que caracteriza al país.

\section{Proceso normativo-institucional de configuración del nuevo escenario}

En Chile existen ciertos antecedentes recientes que son relevantes para el ordenamiento territorial en materia institucional. Dichos antecedentes parten por la creación del Comité de Ministros de la Ciudad y el Territorio, el cual se conformó en el año 2000 a través del instructivo presidencial $\mathrm{N}^{\circ} 011$. A dicho comité se le encargó proponer, supervisar y dirigir el desarrollo de proyectos en zonas urbanas, particularmente aquellos que fuesen emblemáticos para el Bicentenario. El Comité estuvo integrado por los ministros de Vivienda y Urbanismo (quien lo presidía), Secretario General de la Presidencia, Planificación y Cooperación, Educación, Obras Públicas, y Transportes y Telecomunicaciones. Además, solicitaba la presencia permanente de los subsecretarios del Interior, General de la Presidencia, Desarrollo Regional y Administrativo, Marina, Agricultura, Transportes, Telecomunicaciones, los directores de Presupuesto, de la Comisión Nacional de Medio Ambiente (CONAMA), Deportes y Recreación, de la División de Coordinación Interministerial del Ministerio Secretaría General de la Presidencia, el asesor presidencial para la cultura, el secretario ejecutivo de la Comisión de Planificación de Inversiones en Infraestructura y Transporte (SECTRA). Además, se fijó como un órgano de coordinación en temas de desarrollo urbano y ordenamiento territorial, que impulsó proyectos y procesos de reconstrucción del país tras el terremoto del 2010.

En este año, también se crea el Comité de Ministros para la Sustentabilidad, conocido en la actualidad como Consejo de Ministros para la Sustentabilidad (CMS), el cual es presidido por el Ministro del Medio Ambiente e integrado por los Ministros de Agricultura, Hacienda, Salud, Economía, Fomento y Reconstrucción, Energía, Obras Públicas, Vivienda y Urbanismo, Transportes y Telecomunicaciones, Minería y Planificación. Sus funciones han consistido en proponer a la presidencia las políticas para el uso y aprovechamiento sustentable de los recursos naturales, al igual que la definición de criterios de sustentabilidad a considerarse en la elaboración de políticas y procesos que manejan los distintos ministerios. Además, han propuesto la creación de Áreas Protegidas del Estado, incluyendo parques, reservas marinas, santuarios para la naturaleza y áreas marinas costeras. Otra de sus tareas ha sido proponer las políticas sectoriales que deben ser sometidas a procesos de evaluación ambiental estratégica. Además, debe dar sus criterios alrededor para efectuar la participación ciudadana en las Declaraciones de Impacto Ambiental, al igual que sobre los proyectos de ley y actos administrativos que tengan normas de carácter ambiental. 
En el año 2012, se inicia el proceso de elaboración de dos políticas trascendentales para el territorio de Chile. La primera es la Política Nacional de Desarrollo Urbano (PNDU), elaborada por la Comisión Asesora Presidencial que fue conformada especialmente para esta tarea. La Comisión fue capaz de articular un consenso transversal que reunió actores de nivel político, técnico y ciudadano, contando con la participación de especialistas de once ministerios. El objetivo de esta política fue crear una carta que sea capaz de guiar el desarrollo de las ciudades, con énfasis en mejorar la calidad de vida de sus habitantes, descentralización, reorganización institucional, modernización de los cuerpos legales y reglamentarios y finalmente impulsar una mejor convivencia y ambiente propicio para las inversiones en contextos urbanos. La segunda, es la Política Nacional de Desarrollo Rural (PNDR) que estuvo inicialmente bajo la responsabilidad del Comité de Ministros de la Ciudad y el Territorio. Esta política reconoció la diversidad territorial del país, al igual que las características específicas de su geografía y su influencia en los patrones de asentamiento y desarrollo económico. Como consecuencia, reconoció también la heterogeneidad del desarrollo del territorio. En este sentido, propuso un conjunto de lineamientos para orientar y avanzar hacia un desarrollo territorial más integrado del país, promoviendo consigo el bienestar de la población. Ambas políticas, tanto la PNDU como la PNDR fueron elaboradas, promulgándose la PNDU en el año 2014, año en que también se inicia un proceso de revisión y actualización de la PNDR.

En el año 2015 se publica la Política Energética de Chile, la que contó con alta participación ciudadana y que estuvo a cargo de un Comité Consultivo liderado por el Ministro de Energía e integrado por actores claves del sector público, la industria, academia, y la sociedad civil. Esta política, que estaba dentro de la Agenda de Energía, propone una visión a largo plazo (2050) sobre el sector energético, que sería calificado como un sector confiable, sostenible, inclusivo y competitivo, todo con base en un enfoque sistémico. En otras palabras, apunta a avanzar en una energía más sustentable en todas sus dimensiones.

En este año también se crea la Comisión Interministerial de Ciudad, Vivienda y Territorio (COMICIVYT), que entra a reemplazar el Comité de Ministros de la Ciudad y el Territorio. Esta COMICIVYT se planteó como misión servir de ente coordinador de políticas, planes y programas relacionadas con el ordenamiento territorial en su totalidad, incluyendo temáticas relacionadas con infraestructura, calidad de vida y reducción de brechas. Está integrada por los ministros de Vivienda y Urbanismo (quien la preside), Obras Públicas, Transportes y Telecomunicaciones, Bienes Nacionales, Medio Ambiente, Agricultura, Economía, Fomento y Turismo, Minería, Desarrollo Social, Energía, Secretaría General de la Presidencia y por la Subsecretaría de Desarrollo Regional y Administrativo del Ministerio del Interior y Seguridad Pública. Sus tareas han consistido en aportar una visión intersectorial para la coordinación de las inversiones en infraestructura y políticas públicas, modificaciones legales respecto del desarrollo urbano, al igual que proponer políticas de ordenamiento territorial y desarrollo rural y su correspondiente asesoramiento en estos temas. La COMICIVYT inicia en el 2016 la formulación de la Política Nacional de Ordenamiento Territorial (PNOT), al igual que participa en la revisión de la nueva PNDR para su aprobación final.

En síntesis, se puede afirmar que al país le ha costado casi 20 años encaminarse hacia una agenda que progresivamente vaya generando un proceso de construcción de una estructura normativa e institucional para lograr abordar el desafío del ordenamiento territorial. Desde este punto de vista, se advierte una voluntad expresa en el propósito de ordenar y articular los diferentes instrumentos a través de la promulgación de políticas nacionales, particularmente con bajada 
regional en el caso de la PNOT, a través de los PROT. Por lo tanto, el nuevo escenario que plantea la Ley 21.074 para la gobernanza territorial, requiere analizarse bajo ciertos criterios propios de la naturaleza de un sistema de ordenación y planificación territorial integrado.

\section{Enfoque metodológico}

Para el análisis de la incidencia de los Gobiernos Regionales en la gobernanza territorial, el diseño de la presente investigación posee un carácter cualitativo, situándose desde un paradigma interpretativo (Hernández-Sampieri \& Mendoza, 2018), centrándose en la comprensión de los procesos e interrelación de actores institucionales dentro del marco de la gobernanza multinivel, a partir de las reformas en curso. Para tal efecto, se procederá a analizar los instrumentos de planificación de carácter normativo e indicativo de escala intercomunal y regional, considerando aspectos relacionados con la descentralización política, referido a la incidencia de los instrumentos en las políticas públicas sectoriales, así como la interdependencia jerárquica entre ellos. También a aspectos relativos a la descentralización administrativa, referido a la incidencia en la elaboración a aprobación de los instrumentos y regulación sobre usos del suelo. Y, por último, en relación a la descentralización fiscal referido a la vinculación de los instrumentos con partidas presupuestarias.

Las fuentes de información utilizadas para el análisis cualitativo e interpretativo, corresponden a tres fuentes de información: (1) Documentos sobre marco legal o administrativo dispuesto para la aplicación de los instrumentos normativos e indicativos con incidencia en materia de ordenamiento territorial vigentes en Chile; (2) Documentos de trabajo o artículos publicados recientes relacionados con la materia de estudio; (3) Resultados del taller de expertos sobre descentralización regional y transferencia de competencias efectuado en enero 2020 (Orellana \& Orrego, 2020).

\section{Análisis sobre gobernanza territorial de los gobiernos regionales}

La ley 21.074 establece un ajuste a la estructura normativa institucional en materia de gobernanza urbana y territorial para las regiones en Chile y las grandes ciudades que se configuran a partir de dos o más comunas. No obstante, es posible juzgar que esta transición que se espera se consolide una vez que se elija los gobernadores regionales bajo democracia directa programado para el mes de abril 2021, supone un impacto sobre instrumentos de planificación con incidencia en el ordenamiento territorial a escala subnacional, tanto regional como metropolitana.

Este impacto se puede analizar sobre la base de cinco criterios básicos: regulación sobre usos de suelo, vinculación con las decisiones de inversión pública, vinculación con presupuestos, nivel de descentralización política e interdependencia entre instrumentos. Estos criterios, han sido definidos a través de la revisión de variados trabajos de publicación reciente (Vicuña, 2019; Marshall, 2019; Portales, 2019; Arenas \& Orellana, 2019; Horts, 2017). En particular, estos criterios se refieren a: 
- Regulación sobre usos de suelo, referido a la zonificación estricta para establecer una determinación sobre localización e intensidad de las distintas actividades de la población urbana y rural en el territorio.

- Vinculación con las decisiones de inversión pública, referido a la incidencia de las directrices del instrumento en las decisiones respecto a priorización y focalización de las políticas públicas en el territorio.

- Vinculación con presupuesto, referido a la incidencia del instrumento en la cartera de inversión sectorial, regional o local focalizada en el territorio.

- Nivel de descentralización política, referido al nivel de autonomía que tiene la escala de gobierno correspondiente al territorio en la elaboración o modificaciones al instrumento.

- Interdependencia entre instrumentos, referido a la determinación expresa sobre la jerarquía del instrumento respecto a otros que tienen incidencia en el mismo territorio de manera similar o parcial.

Ahora, básicamente a escala subnacional los instrumentos que inciden en el ordenamiento territorial en Chile, son aquellos contemplados en la $\mathrm{LGUC}^{8}$ en el caso de Plan Regulador Intercomunal $(\mathrm{PRI})^{9}$ y el Plan Regional de Desarrollo Urbano (PRDU) ${ }^{10}$, este último vigente en algunas regiones del país, pero pronto a ser reemplazado por los PROT, una vez que se promulgue la Política Nacional de Ordenamiento Territorial (PNOT). Respecto a este último, está contemplado que el PROT se constituya en el principal instrumento de ordenación y planificación a escala regional, sostenida en una política nacional ad hoc. Conforme el artículo 16 de la Ley 21.074 se define el PROT "como un instrumento que orienta la utilización del territorio de la región para lograr su desarrollo sustentable a través de lineamientos estratégicos y una macro zonificación de dicho territorio". Adicionalmente, podemos considerar que la Zonificación del Uso del Borde Costero (ZUBC) constituye otro instrumento de ordenación del territorio sostenido por la Política Nacional de Uso del Borde Costero (Decreto Supremo N475 de 1994), cuyo objetivo es proponer usos preferentes considerando factores geográficos, naturales, recursos existentes, planes de desarrollo, centros poblados próximos o aledaños y definiciones de uso establecidos por organismo competentes. Y, por último, no siendo estrictamente un instrumento de planificación, se puede considerar la Estrategia Regional de Desarrollo (ERD) un instrumento que funciona como un planteamiento global y orientador de las decisiones tanto sectoriales como comunales.

\section{Regulación sobre usos del suelo}

En el caso de la regulación de usos de suelo, con el nuevo escenario existe un fortalecimiento de la gobernanza en ordenamiento territorial para los GORE a partir de lo que define el artículo 18 letra f) de la Ley 21.074 al señalar que en materia de fomento de las actividades productivas, corresponderá al GORE "aprobar los planes regionales de ordenamiento territorial, los planes reguladores metropolitanos e intercomunales y sus respectivos planos de detalle, los planes reguladores comunales, los planes seccionales y los planes de inversiones en infraestructura de movilidad

Ley General de Urbanismo y Construcción, DFL 458

Según Artículo $34^{\circ}$ de la LGUC se entenderá por Planificación Urbana Intercomunal aquella que regula el desarrollo físico de las áreas urbanas y rurales de diversas comunas que, por sus relaciones, se integran en una unidad urbana.

10 El artículo 2.1.5 de la LGCU establece que el PRDU es un instrumento que orienta el desarrollo de los centros urbanos de las regiones. 
y espacio público". Particularmente, referido a la ley $20.958^{11}$ relativa a planes de infraestructura de movilidad y espacio público a escala intercomunal.

Especialmente significativo es el traspaso de la potestad en cuanto a la elaboración y aprobación de los PRI, asumiendo que este constituye el único instrumento estrictamente normativo sobre uso de suelo con incidencia en materia de ordenación del territorio. Lo anterior, porque en el caso del PROT cuando entre en vigencia'2 solo establece en el artículo 17 a) de la Ley 21.074 “con carácter vinculante, condiciones de localización para la disposición de los distintos tipos de residuos y sus sistemas de tratamientos y condiciones para la localización de las infraestructuras y actividades productivas en zonas no comprendidas en la planificación urbanística, junto con la identificación de las áreas para su localización preferente", cuestión implica en general promover directrices sobre el uso de suelo más que normar en forma estricta ${ }^{13}$. Y, por otro lado, la ZUBC tampoco es un instrumento de carácter normativo estricto, sino que solo establece usos preferentes, mientras que el PRDU y Estrategia Regional de Desarrollo (ERD) no tienen incidencia en esta materia en el actual y nuevo escenario, a partir de la implementación de la Ley 21.074.

En síntesis, en cuanto a la regulación sobre usos de suelo, se puede calificar al PROT y la ZBUC con una incidencia mínima en materia de gobernanza en ordenamiento territorial para los GORE, mientras que en el caso de los PRI, aun valorando el traspaso de competencias desde el MINVU a los GORE en su elaboración y aprobación, subsisten una serie de normas de excepción vigentes en la LGUC que limitan la capacidad de normar sobre el uso de suelo a través de los instrumentos de planificación como lo advierte Vicuña (2013), señalando textualmente "a pesar de las restricciones impuestas por algunos planes reguladores comunales para controlar y dirigir la inversión inmobiliaria, aún coexisten formas de regulación urbana centralizadas a través de la LGUC, cuya aplicación está por encima de lo dispuesto en las normativas locales. Dichas regulaciones operan a partir de normas "de excepción", tales como...el Artículo 55, que permite construir vivienda social fuera del límite urbano establecido en los planes reguladores...".14 Por lo tanto, se considera que el PRI en materia de regulación de usos de suelo aportará en forma parcial a la gobernanza territorial de los GORE.

\section{Vinculación con decisiones de inversión pública}

En Chile, las regiones tienen una baja incidencia en la inversión pública que se materializa en sus territorios político-administrativos, siendo principalmente concentrada la toma de decisiones en el gobierno central a través de los ministerios sectoriales, reforzando el carácter centralista y unitario de nuestro país a pesar de las reformas en curso. Conforme un informe publicado por la OECD-SUBDERE (2013) afirma que Chile está entre los países más centralizados de la organi-

Ley promulgada el año 2016, que Establece un Sistema de Aportes al Espacio Público modificando la LGUC

A la fecha de envío de este trabajo, la PNOT está en trámite en la Contraloría General de la República (CGR) para su aprobación, por lo tanto, también pendiente el diseño de un reglamento para elaboración de los PROT que sea aprobado por la COMICIVYT

13 En particular, vale la pena señalar que en el marco de elaboración de la PNOT fue recurrente el debate sobre la conveniencia y factibilidad de hacer de los PROT un instrumento normativo de mayor escala, particularmente por la complejidad que significa sobreponer un instrumento de escala regional sobre un PRI que en el caso de la Región Metropolitana con el Plan Regulador Metropolitano de Santiago (PRMS) se traslapa en escala, así como en el caso de la Región de Coquimbo donde existe un PRI para cada una de las tres Provincias que la configuran.

14 En un estudio reciente efectuado por los autores de esta publicación sobre el proceso de metropolización en la ciudad de Los Ángeles, se pudo comprobar que la superficie urbanizada fuera de los límites urbanos impuestos por su Plan Regulador Comunal prácticamente era equivalente en superficie al espacio regulado por el instrumento, básicamente a través de la aplicación del artículo 55 de la LGUC. 
zación, junto a Grecia e Irlanda, considerando que el gasto subnacional representó solo un 3,0\% del PIB y un $13,1 \%$ de los gastos públicos, lo que contrasta con el promedio de la OCDE que es del $16,6 \%$ y del $40,2 \%$, respectivamente. Así mismo, en otro estudio publicado por Horts (2018), considerando solo países unitarios, se observa que mientras Dinamarca y Suecia corresponden a los países más descentralizados, Irlanda y Grecia aparecen como los más centralizados, ubicándose Chile entre 26 países en el lugar 18 en términos de nivel de centralización.

Considerando la situación descrita, el nuevo escenario que plantea la ley 21.074 en materia de descentralización en las decisiones de inversión pública de promulgarse la PNOT y hacerse efectivo el PROT, es el instrumento que entrega la mayor posibilidad a los GORE para incidir en el ordenamiento territorial. Y, más aún, considerando que en el mismo numeral de la ley se señala que "el plan regional de ordenamiento territorial será de cumplimiento obligatorio para los ministerios y servicios públicos que operen en la región y no podrá regular materias que tengan un ámbito de influencia u operación que exceda del territorio regional ni áreas que estén sometidas a planificación urbanística". No obstante, sobre esto último resulta todavía ambiguo el cómo se aplicará un PROT a regiones como la Metropolitana y la Región de Coquimbo donde el Plan Regulador Metropolitano de Santiago (PRMS) y los tres PRI, respectivamente, cubren todo el territorio regional.

Conforme lo anterior, se puede calificar la incidencia como parcial, dado que la misma ley señala en el artículo 17 letra a) que los GORE podrán "elaborar y aprobar el plan regional de ordenamiento territorial en coherencia con la estrategia regional de desarrollo y la política nacional de ordenamiento territorial, previo informe favorable de los ministros que conforman la Comisión Interministerial de Ciudad, Vivienda y Territorio", lo cual determina a priori todavía la aprobación de parte de instancias interministeriales dependientes del gobierno central.

\section{Vinculación con presupuestos}

El carácter no prospectivo del PRI probablemente explica su nula vinculación directa con presupuestos públicos, salvo de manera indirecta porque determina condicionamientos en uso de suelo para la localización de infraestructuras, equipamientos y edificaciones varias. La razón fundamental descansa en el hecho que este tipo de instrumento tiene un carácter más regulatorio que de planificación, además no está ligado a un plan estratégico a escala de ciudad intermedia o metropolitana, ya que legalmente este tipo de instrumento no se encuentra contemplado en la legislación chilena.

Si bien los PRI debieran contemplar los lineamientos vigentes de la ERD para su actualización o modificaciones parciales, no existe en la LGUC obligación alguna de hacerlo. Adicionalmente, la ERD si bien supone una carta de navegación para los GORE de carácter prospectivo con horizontes que van de 10 a 15 años, en general, tienen una incidencia mínima respecto a las decisiones de inversión pública de ministerios sectoriales. A esto se suma, el hecho que la mayor proporción del presupuesto de los GORE se incluye en el Fondo Nacional de Desarrollo Regional (FNDR) ${ }^{15}$ que constituye un $44 \%$ del total (DIPRES, 2017), presupuesto que financia proyectos como un complemento al financiamiento municipal, más que recursos económicos vinculados directamente a los

FNDR es "un programa de inversiones públicas, con fines de compensación territorial, destinado al financiamiento de acciones en los distintos ámbitos de infraestructura social y económica de la región, con el objetivo de obtener un desarrollo territorial armónico y equitativo" 
instrumentos de planificación a escala regional o intercomunal, que incidan en el ordenamiento territorial de la región.

En cuanto a los PROT, se presume que el espíritu de la ley al dotar a los GORE de este instrumento, constituye un avance significativo en aras de lograr fortalecer en la región los procesos de ordenación del territorio, para lo cual el carácter vinculante que establece que será, como ya se señaló, de cumplimiento obligatorio para los ministerios y servicios públicos que operen en la región, no implica necesariamente que esté vinculado a un presupuesto específico. No obstante, se plantea en al artículo 20 bis de la ley 21.074 que "Las funciones generales y de ordenamiento territorial, de fomento de las actividades productivas y de desarrollo social y cultural, incluidas aquellas que se ejerzan en virtud de una transferencia de competencia, serán ejercidas en forma coherente con las políticas públicas nacionales vigentes, correspondiendo al ministro respectivo velar por aquello", por lo cual no queda totalmente claro, si será bajo descentralización administrativa y/o fiscal y menos aún si el PROT no tendrá vinculación con presupuesto alguno.

Por último, para el caso del PRDU, la consecución de convenios de programación debieron ser el mecanismo principal para focalizar políticas públicas que mejoren la articulación entre los centros urbanos, no obstante, en la práctica se han remitido a acuerdos entre un ministerio sectorial y el GORE que se traduce solo en infraestructura, pero con una incidencia mínima en los presupuestos que comprometen la gobernanza en ordenamiento territorial. Y, finalmente, la ZUBC constituye un ejercicio de planificación acotada que tiene una nula incidencia en los presupuestos orientados a temas de ordenación del territorio. En definitiva, como lo señala Vicuña (2019), en su estudio de planificación urbana integrada en áreas metropolitanas comparadas, debe haber una integración decisional donde se exprese la capacidad de un sistema de planificación de vincularse con la inversión pública o que la sujeción de la decisión de inversión está determinada por los objetivos perseguidos por los instrumentos de planificación.

\section{Nivel de descentralización política}

Resulta significativo que bajo este criterio la mayoría de los instrumentos se refuerzan en materia de descentralización política para incidir en la gobernanza en ordenamiento territorial, especialmente el PRI, PRDU y el PROT. En el caso del PRI, ya no será necesario obtener la aprobación del MINVU para actualizar o modificar este instrumento, como ha sido hasta ahora, tal como se expresa en el artículo 18 letra f) de la Ley 21.074 antes señalado. Se reafirma lo anterior, cuando se establece en la misma ley en el artículo 37, el reemplazo de la frase "Los Planes Reguladores Intercomunales serán aprobados por decreto supremo del Ministerio de Vivienda y Urbanismo, dictado por orden del Presidente de la República, previa autorización del Intendente respectivo", por la siguiente: "Los Planes Reguladores Intercomunales o Metropolitanos serán aprobados por el consejo regional y promulgados por resolución del gobernador regional".

Así mismo, si se mantiene el PRDU como instrumento de planificación de carácter indicativo conviviendo con el PROT, bajo el mismo artículo 18 letra f) de la Ley 21.074 es posible interpretar que también será resorte del GORE aprobarlo sin intermediar la decisión con el MINVU u otra entidad del gobierno central. En síntesis, en ambos casos la incidencia bajo este criterio será total para el GORE. Mientras que en el caso del PROT, se señala en la Ley 21.074 en el artículo a) que los GORE deberán "Elaborar y aprobar el plan regional de ordenamiento territorial en coherencia 
con la estrategia regional de desarrollo y la política nacional de ordenamiento territorial, previo informe favorable de los ministros que conforman la Comisión Interministerial de Ciudad, Vivienda y Territorio", cuestión que permite sancionar el criterio de descentralización política solo en término parcial.

Y, en cuanto a la ZUBC, esta deberá ser respetada por el PROT e incorporada en la macro-zonificación. Ahora, en el caso de que no haya en una región resolución al respecto, entonces se señala en el artículo 17 a) de la ley 21.074 que "el gobierno regional deberá proponer un proyecto de zonificación del borde costero de la región, así como las eventuales modificaciones a la zonificación vigente, en concordancia con la política nacional existente en la materia. Dicha zonificación deberá ser aprobada mediante decreto supremo expedido a través del Ministerio de Defensa Nacional y será reconocida en el respectivo plan regional de ordenamiento territorial", por eso se considera para este criterio una incidencia mínima.

$\mathrm{Y}$, finalmente, en el caso de la ERD tanto antes como bajo el nuevo escenario que plantea la Ley 21.074 en materia de gobernanza territorial para los GORE, no cambia sustantivamente la situación en cuanto a la incidencia en la elaboración y aprobación de este instrumento que se puede calificar como total, sin embargo, en la práctica, es quizás el único instrumento con menor impacto en materia de ordenamiento territorial.

\section{Interdependencia entre instrumentos}

Cualquier sistema de ordenación y planificación urbana y territorial, precisa que los instrumentos normativos e indicativos sostengan una integración articulación de carácter multiescalar. Para el caso de Chile, los instrumentos que inciden en la ordenación del territorio a escala regional, metropolitana, intercomunal y comunal no estaban debidamente integrados e interdependientes antes de la promulgación de la ley 21.074, hecho que esta ley solo corrige en forma parcial.

La razón de lo anterior, obedece al hecho qué en el caso del PRI, este instrumento está integrado y tiene interdependencia con cada Plan Regulador Comunal (PRC) de aquel conjunto de comunas que configuran una inter comuna o un área metropolitana, tal como en los casos de Santiago, Valparaíso y Concepción. Ambos instrumentos y sus normativas que los vinculan, se sostienen en diversos articulados contenidos en la LGUC y es en rigor la única interdependencia entre instrumentos que inciden en la gobernanza en ordenamiento territorial, por eso se califica como Parcial. Sin embargo, en el caso del PRDU siendo un instrumento que igual también lo sostiene la LGUC, no tiene una estrecha vinculación con los PRI o PRC en las regiones que está vigente, ni siquiera con la ERD, cuestión que implica que se desaprovecha su carácter más estratégico interurbano, por eso se califica como mínima su incidencia.

Por su parte, una vez que se promulgue la PNOT y se reglamente los PROT, al menos se establece que este instrumento deberá ser considerado por los instrumentos normativos como el PRI y los PRC por su mayor jerarquía a escala subnacional. Particularmente, en relación a los usos preferentes según lo que se define en el artículo 17 a) de la ley 21.074, donde se señala que "también establecerá, con carácter vinculante, condiciones de localización para la disposición de los distintos tipos de residuos y sus sistemas de tratamientos y condiciones para la localización de las 
infraestructuras y actividades productivas en zonas no comprendidas en la planificación urbanística, junto con la identificación de las áreas para su localización preferente".

Adicionalmente, no se establece con claridad la jerarquía del PRI, PRC o del PROT respecto a la ZUBC, donde en este último caso según lo que se señala en al artículo 17 a) de la ley 21.074 se apunta sobre el PROT más a cómo proceder cuando está declarada legalmente una ZUBC y cuando no está declarada. Incluso, tampoco hay definiciones claras sobre la interdependencia entre los PRI o PRC respecto a la ZUBC en el propio Decreto Supremo $475 \mathrm{ni}$ en la ley LGUC, sin mencionar que no está determinado tampoco como la ERD es considerada en la ZUBC, por eso para instrumento se considera su incidencia mínima en cuanto al criterio de interdependencia entre instrumentos.

Finalmente, a propósito de la ERD, la consideración que tuvo la redacción de la ley 21.074 en el encabezado de su artículo 17 a) al señalar que los GORE deberán "elaborar y aprobar el plan regional de ordenamiento territorial en coherencia con la estrategia regional de desarrollo y la política nacional de ordenamiento territorial", sin duda significa un avance importante en términos de normar la interdependencia de un instrumento estrictamente indicativo como la ERD, no vinculante, con uno también indicativo pero vinculante con la política pública. A pesar de ello, dada la casi nula interdependencia formal con los PRI, PRDU y ZUBC, solo admite calificar la incidencia de la ERD en un nivel mínimo.

\section{Síntesis de resultados}

Los resultados del análisis interpretativo sobre la gobernanza territorial que podrán ejercer en el nuevo escenario normativo-institucional los GORE, al alero que le otorga la ley 21.073 y especialmente la ley 21.074, muestran ser insuficiente para corregir las brechas de inequidad socio territorial existentes en el país entre regiones y al interior de las propias regiones. Lo anterior, refuerza el juicio de la OECD-SUBDERE (2017) en el sentido que "la planificación coordinada se ve limitada por un proceso presupuestario y mecanismos de financiación que no favorecen la coherencia entre los instrumentos de planificación. Las estrategias regionales y locales no están directamente vinculadas a fuentes de financiamiento, y su implementación está atada a la capacidad anual de los presupuestos regionales, que depende de transferencias desde el nivel central. El financiamiento subnacional sigue una lógica sectorial y los proyectos que componen una iniciativa integrada se evalúan de forma independiente, sin referencia a un plan maestro" (OECD-SUBDERE, 2017:61).

EI PROT mejora en forma importante la capacidad de articular la planificación territorial a escala intercomunal y regional, otorgando un mayor peso específico a la ERD como carta de navegación, en un contexto legitimado políticamente, a partir de la elección de los Gobernadores Regionales proyectada para abril de 2021, así como también fortalece la capacidad de los GORE para orientar la política pública en el territorio. Sin embargo, su aprobación depende o queda sujeta a una instancia interministerial como la COMICIVYT, que representa el nivel central de gobierno, más allá del traspaso de competencias a los GORE que pudiera sustentar una mayor autonomía, quedando a merced de la voluntad de los ministerios y la venia del Ministerio de Hacienda, si compromete transferencias de recursos fiscales. La ley tampoco es clara respecto de si 
cada ministerio que integra la citada comisión actúa para estos efectos en conjunto con los otros miembros o lo hace separadamente.

Finalmente, en cuanto a la interdependencia con el resto de los instrumentos de planificación que inciden en el ordenamiento territorial, particularmente en el PRI, hay un avance porque se logra alcanzar mayor incidencia de los GORE en la medida que deberán considerar las directrices de los PROT. Sin embargo, la elaboración del PRI seguirá dependiendo de la Seremi MINVU y con la aprobación de parte del GORE. Adicionalmente, la LGUC sostiene todavía un número importantes de articulados que promueven normas de excepción que debilitan el alcance de los $\mathrm{PRI}$, donde la resolución escapa muchas veces a una potestad regional. Y, por último, en el caso de la ZUBC, se presentan vacíos importantes sobre su relación jerárquica respecto a los PROT, ya que no es precisa la ley 21.074 en cuanto al procedimiento en el caso de las regiones que tengan promulgada su ZUBC, respecto de los casos donde esto no ocurre, mientras que en lo relacionado con el PRDU, no hay mención alguna sobre cómo se articulará este con el PROT, ni tampoco claridad respecto a la convivencia entre ambos instrumentos de naturaleza similar. El supuesto es que estos últimos sustituirán a los primeros.

\section{Conclusiones}

La diversidad geográfica existente en Chile también exige respuestas diferenciadas, desde una mirada territorial que predomine por sobre la mirada sectorial (que se expresa preferentemente a escala nacional). Esto puede ser considerado una materia pendiente, ya que no hay ningún reflejo de ello ni en la estructura institucional, ni en los dispositivos ligados a la planificación territorial, ni en lógicas de uso del suelo, como tampoco en los niveles de descentralización requeridos para un proceso de toma de decisiones sensible a las características descritas.

El resultado de esta combinación entre las condiciones impuestas por la base físico-natural y la respuesta de la sociedad en torno a cómo organizar en el territorio, las inversiones, las distintas actividades, la infraestructura y los asentamientos, es la generación de disparidades territoriales que en algunos casos alcanzan niveles inaceptables. Por la profundidad con que se expresan algunas de estas disparidades en el territorio, debiéramos intervenir no solo estableciendo una visión territorial y reglas del juego que permitan mejores resultados, sino además instaurando instrumentos de planificación territorial que incorporen de manera más efectiva la influencia de la geografía ya descrita, allí donde las disparidades alcancen una dimensión preocupante, con el objeto de diseñar y poner en marcha verdaderos mecanismos de compensación y de solidaridad territorial, hasta ahora casi inexistentes en el país.

Del mismo modo, dada a diversidad mencionada se requiere de una capacidad de respuesta a distintas escalas, no excluyentes, que eviten la estandarización de los problemas y de las soluciones, dando paso a una mejor articulación entre sectores, pero sobre todo entre los niveles de intervención nacional, regional y local, como estructuras independientes pero interconectadas. Es por ello que los desafíos de una gobernanza territorial a la escala regional suponen no sólo un enfoque consensuado, sino también atribuciones y competencias suficientes para este nivel, sobre cuya base se puede generar una planificar y gestión territorial integrada. 
El proceso de fortalecimiento y descentralización regional impulsado en Chile en estos últimos diez años constituye un avance significativo en la dirección señalada, después de décadas de resistencia hacia procesos que garanticen mayores niveles de descentralización política, administrativa y, en menor medida, fiscal, en favor de las regiones. Sin embargo, resultan insuficientes al menos para que en materia de gobernanza territorial el nuevo escenario sea más favorable, para que las directrices estratégicas que se promueven desde la ERD y desde una futura PNOT, sean debidamente sostenidas por los instrumentos de planificación de escala intercomunal y regional actuales $y$, especialmente por los futuros PROT, en lo que se espera sea el inicio de un nuevo trato Estado-Región, al estar dotadas estas últimas por primera vez, de un instrumento de carácter propiamente territorial, que tendrá aspectos vinculantes para una parte de estructura pública del país.

\section{Referencias bibliográficas}

AGHÓN, G. \& CORTÉS, P. Descentralización y gobiernos municipales en América Latina. En: R. Jordán y D. Simioni (Comps.). Ciudades intermedias de América Latina y el Caribe: propuestas para la gestión urbana. Santiago de Chile: Comisión Económica para América Latina y el Caribe (CEPAL), 1998, (LC /L .1117)

AMIN, A. \& THRIFT, N. Institutional issues for the European regions: fron markets and plans to socioeconomics and power associations. Economy and Society, 1995, №24, p.41-61

ARENAS, F. El territorio chileno, un espacio en (de)formación: el difícil balance entre Estado unitario y una necesaria autonomía regional. Chile mirando hacia su futuro. En C. Auroi y C. Sáez Muñoz (Coeds.). La Revista, Boletín № 77, 2016-17, Sociedad Suiza de Americanistas (SSA-SAG),. Septiembre 2017, pp. 114-123.

ARENAS, F. La construcción de las regiones chilenas: desde los hitos históricos hasta los desafíos actuales. En H. Von Baer (Ed.). Pensando Chile desde sus Regiones. Temuco: Agrupación de Universidades Regionales, Red Sinergia Regional, Ediciones Universidad de La Frontera, 2009, p. 61-76.

ARENAS, F. \& ORELLANA, A. Aportes desde el ordenamiento territorial a la descentralización política y administrativa del país. En H. Von Baer \& N. Bravo (Eds.). Desarrollo territorial colaborativo; Descentralización, poder, competencias y recursos. Temuco: Ediciones Universidad de la Frontera, 2019, p. 153-160

BALBONTÍN, R., ESCOBAR, M. \& SEEMANN, A. Financiamiento de los gobiernos regionales en Chile. Santiago de Chile: Serie de Estudios de Finanzas Públicas de la Dirección de Presupuestos, Ministerio de Hacienda de Chile, 2017.

BRENNER, N. Globalization as reterritorialisasion. The re-scaling on urban governance in the European Union. Urban Studies, 1999, N³6, p. 351-451

CAMARA, G. La organización territorial de España. Una reflexión sobre el estado de la cuestión y claves para la reforma constitucional. Revista de Derecho Político, N0101, enero-abril 2018, p. 395-430. 
CEPAL. Panorama del desarrollo territorial en América Latina y el Caribe; Agendas globales de desarrollo y planificación multinivel. Nueva York: Documentos de proyecto, 2017.

CERECEDA, P. \& ERRÁZURIZ, A. Geografía de Chile Ilustrada. Ecogeografía. Santiago: Instituto de Geografía de la Pontificia Universidad Católica de Chile, ZIG-ZAG, 2005.

FERNÁNDEZ, V.R., AMIN, A. \& VIGIL, J.I. (Comps.). Repensando en el desarrollo regional; Contribuciones globales para una estrategia latinoamericana. Buenos Aires: Miño \& Dávila, 2008.

FERRAO, J. O Ordenamiento do território como política pública. Lisboa: Fundación Calouste Gulbenkian, 2011.

GALLAND, D. \& HARRISON, J. Conceptualising Metropolitan Regions: How Institutions, Policies, Spatial Imaginaries and Planning Are Influencing Metropolitan Development. In K. Zimmermann, D. Galland \& J. HARRISON (Eds.). Metropolitan Regions, Planning and Governance. Berlín: Springer, 2019, p. 1-22.

HERNÁNDEZ-SAMPIERI, R. \& MENDOZA, C. Metodología de Investigación, las rutas cuantitativa, cualitativa y mixta. Ciudad de México: Editorial McGraw Hill, 2018.

HERNÁNDEZ, Y. El ordenamiento territorial y su construcción social en Colombia: ¿un instrumento para el desarrollo sustentable? Cuadernos de Geografía, Revista Colombiana de Geografía, N019, 2010, p. 97-109

HORTS, B. Descentralización Fiscal: Antecedentes para una reforma en Chile en materia de financiamiento regional. Santiago de Chile: Serie Informe Económico 273, Libertad y Desarrollo, 2018.

MARSHALL, C. Centralismo y formas fluidas de planificación territorial en Chile: mecanismos de gobernanza horizontal para la agenda urbana local. En Anales de Arquitectura (2018-2019). Santiago de Chile: ARQ Ediciones, 2020, p.130-139.

MARSHALL, C. La dicotomía de los procesos de descentralización administrativa y planificación urbano-regional en Chile; efectos sobre la planificación y gestión subnacional. Tesis de doctorado. Facultad de Arquitectura, Diseño y Estudios Urbanos, Pontificia Universidad Católica de Chile, 2018.

OECD-SUBDERE (2017). Revisión de Gobernabilidad Multinivel en Chile: Modernización del Sistema Municipal, Conclusiones principales y recomendaciones. Recuperado el 15 de marzo 2019: file:/// Users/mimac/Downloads/Chile-multi-level-main-findings-ES\%20(1).pdf

ORELLANA, A. \& MARSHALL, C. Análisis de la complejidad intercomunal para el desarrollo de las áreas metropolitanas en Chile. En A. Orellana, C. Miralles-Guasch \& L. Fuentes (Eds.). Las Escalas de la Metrópolis: Lejanía versus proximidad. Santiago de Chile: Colección Estudios Urbanos UC, RIL Editores, 2019, p. 251-280 
ORELLANA, A. \& ORREGO, C. Descentralización regional, gobernadores electos y transferencia de competencias. Santiago de Chile: Documento de Trabajo IEUT No9, 2020.

ORELLANA, A. \& MARSHALL, C. La relación entre inversión municipal pública y calidad de vida en las ciudades metropolitanas en Chile. Cadernos Metrópole, 2017, Vol. 19, º39, p. 665-686.

ORELLANA, A., BANNEN, P., FUENTES, L., GILABERT, H. \& PAPE, K. Huellas del proceso de metropolización en Chile. INVI, Vol. 28, N77, p. 17-66.

PORTALES, D. La co-construcción de la descentralización y el desarrollo territorial: requisito indispensable para el buen avance del proceso. Aportes desde el ordenamiento territorial a la descentralización política y administrativa del país. En H. Von Baer \& N. Bravo (Eds.). Desarrollo territorial colaborativo; Descentralización, poder, competencias y recursos. Temuco: Ediciones Universidad de la Frontera, 2019, p. 161-179.

PRATS, J. Chile será descentralizado o no será desarrollado. En H. Von Baer (Ed.). Pensando en Chile desde sus regiones. Temuco: Ediciones Universidad de la Frontera, 2009, p. 11-18.

REHREN, A., ORELLANA, A., ARENAS, F. \& HIDALGO, R. La regionalización en un contexto de urbanización regional: desde los desafíos a las propuestas de criterios de zonificación para el caso chileno. Revista de Geografía Norte Grande, 2018, N69, p. 191-209

RONDINELLI, D. Government Decentralization in Comparative Perspective: Theory and Practice in Developing Countries. International Review of Administrative Sciences, 1981, Vol. 47, N 2, p. 133-145.

SÁNCHEZ, M.T., BOCCO, G. \& J. CASADO, M. La política de ordenamiento territorial en México: de la teoría a la práctica. México D.F.: Instituto de Geografía, Centro de Investigaciones en Geografía Ambiental, UNAM, Secretaría de Medio Ambiente y Recursos Naturales (SEMARNAT), Instituto Nacional de Ecología y Cambio Climático, 2013.

SAGREDO, R., GONZÁLEZ, J. \& COMPAN, J. Atlas histórico de las divisiones político-administrativas de Chile 1810-1940. Santiago de Chile: Instituto Geográfico Militar, Pontificia Universidad Católica de Chile y Dirección de Bibliotecas, Archivos y Museos, 2016.

VICUÑA, M. El marco regulatorio en el contexto de la gestión empresarialista y la mercantilización del desarrollo urbano del Gran Santiago, Chile. INVI, 2013, Vol. 28, N078, p. 181-219.

VICUÑA, M. Dimensiones de la planificación urbana integrada: claves y desafíos para la planificación de las áreas metropolitanas. En A. Orellana, C. Miralles-Guasch \& L. Fuentes (Eds.). Las Escalas de la Metrópolis: Lejanía versus proximidad. Santiago de Chile: Colección Estudios Urbanos UC, RIL Editores, 2019, p. 185-220 


\section{Legislación Consultada}

Decreto Ley 575 de 1974, Regionalización del País. Diario Oficial de la República, Santiago de Chile, 13 de julio de 1974.

Decreto Fuerza Ley 458 de 1976, Ley General de Urbanismo y Construcciones, Diario Oficial de la República, Santiago de Chile, 13 de abril de 1976.

Decreto Supremo N475 de 1994, Ley Establece Política Nacional de Uso del Borde Costero del litoral de la República y crea Comisión Nacional, 14 de diciembre de 1994.

Ley 19.175, 1993, Ley Orgánica Constitucional sobre Gobierno y Administración Regional. Diario Oficial de la República de Chile, 20 de marzo de 1993.

Ley 21.073 de 2018, Regula la elección de gobernadores regionales y realiza adecuaciones a diversos cuerpos legales. Diario Oficial de la República, 1 de marzo de 2018.

Ley 21.074 de 2018, Fortalecimiento de la Regionalización del País. Diario Oficial de la República, 15 de febrero de 2018. 
